\title{
Total atrial paralysis after permanent dual chamber cardiac pacing
}

\author{
Iain A Simpson, Sue Jones, A John Camm
}

\begin{abstract}
A 73 year old woman with known ischaemic heart disease and ischaemic ventricular tachycardia underwent temporary dual chamber pacing as part of the treatment to suppress tachycardia. Insertion of a permanent dual chamber system seven days later was uncomplicated. Total atrial paralysis, with inability to pace the atrium or obtain an atrial electrocardiogram from four atrial sites, developed within 24 hours of the permanent system being implanted. Atrial electrical and mechanical function recovered over several weeks.
\end{abstract}

(Br Heart J 1993;69:191-192)

Atrial paralysis is a rare but well recognised abnormality. It has been reported as a permanent feature in childhood as a familial abnormality, ${ }^{1}$ as a cardiac manifestation of fascioscapulohumeral muscular dystrophy, ${ }^{2}$ after myocarditis, ${ }^{3}$ or associated with right ventricular dysplasia ${ }^{4}$ or Ebstein's anomaly. ${ }^{5}$ Transient atrial paralysis has also been reported after intravenous flecainide ${ }^{6}$ or early after cardiac surgery. ${ }^{7}$ Permanent dual chamber cardiac pacing is an established treatment for atrioventricular conduction disorders with or without sinus node dysfunction. Such pacing is also widely used for tachycardia suppression, as an adjunct to pharmacological treatment. Though dual chamber pacing is popular total atrial paralysis is rare. We report the unusual case of the rapid development of total atrial paralysis after insertion of a permanent dual chamber system in a patient who had satisfactory spontaneous atrial electrical activity and excellent atrial pacing thresholds at the time of implantation.

\section{Case report}

A 73 year old woman presented with a diagnosis of ischaemic ventricular tachycardia after an anterior myocardial infarction two years before. She presented to hospital with sustained uniform recurrent ventricular tachycardia (cycle length $260 \mathrm{~ms}$ ) that required DC cardioversion and was resistant to treatment with lignocaine, procainamide, flecainide, and bretylium tosylate. Tachycardia was initially controlled by amiodarone but she was readmitted with recurrent ventricular tachycardia a month later. Further combinations of treatment were tried. Only amiodarone plus sotalol in combination with atrial pacing produced satisfactory tachycardia suppression. Dual chamber permanent pacing was used to manage treatment-induced symptomatic bradycardia and for tachycardia suppression. An Intermedics Cosmos II bipolar pacemaker was inserted without difficulty. The atrial electrogram showed a $P$ wave of $1.5 \mathrm{mV}$ with an atrial pacing threshold of $0.9 \mathrm{~V}$ at $0.5 \mathrm{~ms}$ (impedance $440 \Omega$ ) (fig 1) and atrial mechanical activity visualised on $x$ ray screening. At a pacing check the next day no atrial activity was visible on the resting electrocardiogram and it was not possible to capture the atrium with the maximum
Department of Cardiological Sciences, St George's Hospital Medical School, London I A Simpson $S$ Jones

A J Camm

Correspondence to: Dr I A Simpson MD Wessex Regional Cardiac Unit, Southampton General Hospital, Tremona Road, Southampton SO9 $4 \mathrm{XY}$.

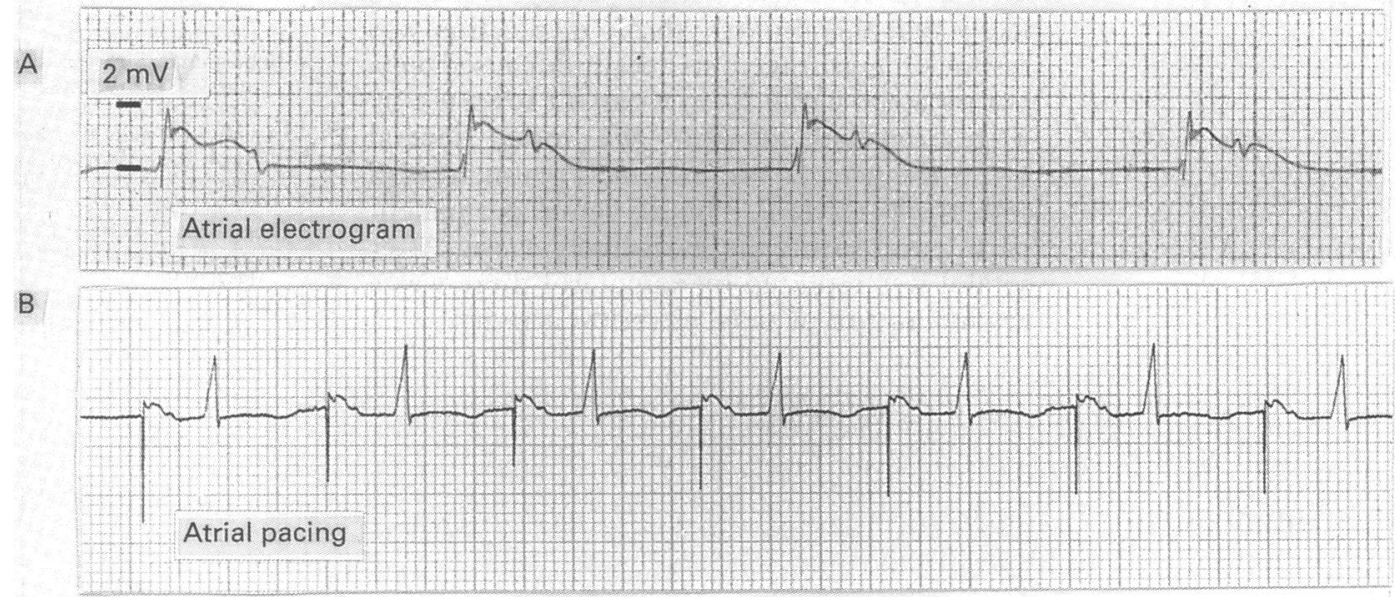

Figure 1 Atrial recordings obtained during permanent pacemaker implantation. (A) Atrial electrogram recorded via the atrial electrode. The $P$ wave amplitude was $1.5 \mathrm{mV}$. (B) Atrial pacing via the implanted atrial electrode showing a stimulated $P$ wave. The atrial pacing threshold at the time of implantation was $0.9 \mathrm{~V}$ at $0.5 \mathrm{~ms}$. 


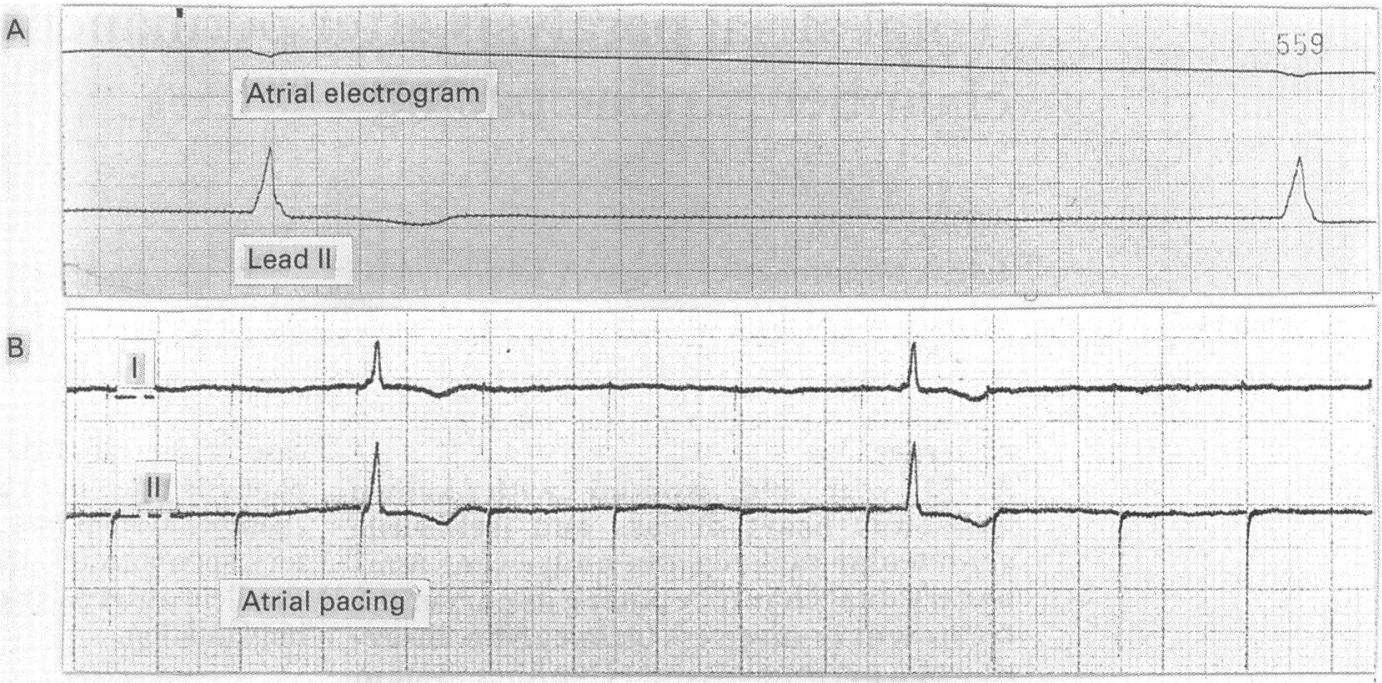

Figure 2 Atrial recordings obtained at the time of re-exploration the day after implantation of the permanent pacemaker. (A) No spontaneous atrial activity could be recorded from the atrial electrode from any of four different sites within the atrium. (B) Atrial pacing spikes were evident but atrial stimulation was not possible despite pacing at $10 \mathrm{~V}$ with a pulse duration of $1 \mathrm{~ms}$.

atrial output of the pacemaker. $x$ Ray screening showed that the atrial lead was not displaced, but there was no evidence of atrial mechanical activity. It was not possible to pace the atrium at four different atrial sites at up to $10 \mathrm{~V}$ and no atrial electrogram could be recorded (fig 2). Manipulation of the atrial lead into the right ventricle allowed ventricular pacing at a threshold below $0.5 \mathrm{~V}$. This confirmed the diagnosis of total atrial paralysis. The atrial lead was left in situ and the pacemaker was reprogrammed to the VVI mode. Follow up 10 days later showed no evidence of atrial electrical activity from an atrial electrogram recorded via the pacemaker options.

\section{Discussion}

In this patient total atrial paralysis developed within 24 hours of permanent dual chamber pacing. Unless the exact site of pacing was a factor in the development of atrial paralysis, its onset cannot solely be related to atrial pacing because temporary atrial pacing was performed for a week before permanent pacing. It is more likely that the antiarrhythmic medication was responsible. Intravenous flecainide caused temporary atrial paralysis in a patient reported by Tognarini and Pardini. ${ }^{6}$ We tested several potential agents in our patient. Treatment with sotalol was started 24 hours before permanent pacing and seems the most likely cause. After sotalol was replaced by xamoterol there was a gradual return of atrial electrical activity.
Total atrial paralysis should be considered after permanent dual chamber pacing in any patient in whom failure of atrial capture is associated with absence of atrial depolarisation and absent mechanical atrial activity on $x$ ray screening or echocardiography, particularly if the patient is receiving antiarrhythmic drugs. The course in these patients is unknown but if drug treatment is implicated, one might reasonably expect a return of atrial electrical and mechanical activity after treatment ends. The presence of atrial paralysis during temporary atrial pacing may be an indication for permanent rate-responsive ventricular pacing rather than dual chamber pacing.

1 Ward DE, Ho SY, Shinebourne EA. Familial atrial standstil and inexcitability in childhood. Am J Cardiol 1984;53 965-7.

2 Stevenson WG, Perloff JK, Weiss JN, Anderson TL. Fascioscapulohumeral muscular dystrophy: evidence for selective, genetic electrophysiologic cardiac involvement. J Am Coll Cardiol 1990;15:292-9.

3 Talwar KK, Radhakrishnan S, Chopra P. Myocarditis manifesting as persistent atrial standstill. Int J Cardio 1988;20:283-6.

4 Belhassen B, Shapira I, Hammerman C. Unusual manifestations of arrhythmogenic right ventricular dysplasia as ventricular fibrillation, atrial paralysis, and hypoexcitable ventricular fibrillation, atrial paralysis, and
right ventricle. $B r$ Heart $J$ 1988;59:263-5.

5 Pierard LA, Henrard L, Demoulin JC. Persistent atrial standstill in familial Ebstein's anomaly. Br Heart J 1985; 53:594-7.

6 Tognarini $S$, Pardini E. A case of atrial paralysis during the use of intravenous flecainide acetate. Cardiologia 1989; 34:725-9.

7 Ruff P, Leier CV, Schaal SF. Temporary atrial standstill. Am Heart J 1979;98:413-20. 